\title{
Trajectory Planning for Smooth Transition of a Biped Robot
}

\author{
Zhe Tang ${ }^{1,2}$, Changjiu Zhou ${ }^{2}$, Zenqi Sun ${ }^{1}$ \\ ${ }^{1}$ Department of Computer Science and Technology \\ Tsinghua University \\ Beijing 100084, P.R. China \\ ${ }^{2}$ School of Electrical and Electronic Engineering \\ Singapore Polytechnic \\ 500 Dover Road, Singapore 139651 \\ www.robo-erectus.org
}

\begin{abstract}
This paper presents a third-order spline interpolation based trajectory planning method which is aiming to achieve smooth biped swing leg trajectory by reducing the instant velocity change which occurs at the time of collision of the biped swing leg with the ground. We first characterize the bipedal walking cycle and point out some major issues that need to be addressed to plan a continuous swing leg trajectory by using the concept of the spline interpolation and zero moment points (ZMP). We demonstrate that the impact effects can be avoided at the time of the swing foot's heel touching with the ground. The proposed biped trajectory planning method has been tested on our soccer-playing humanoid robot, Robo-Erectus, which got Second Place at the 2002 RoboCup Humanoid Walk competition.
\end{abstract}

\section{INDRODUCTION}

Trajectory planning is crucial for a stable motion of biped robots $[5,10,11]$. In particular some basic constraints, such as existence of the solution for inverse kinematics of legs, limitation of joint angle range, limitation of joint angle velocity, collision between links and so on, have to be satisfied through out the entire gait cycle. However the trajectories are not uniquely defined by these constraints. To determine the most suitable trajectory in an optimization, we have to select some possible cost functions, e.g. the energy consumption, the global stability and etc.

Stability is a basic requirement of gait generation for the biped robot. There are two measurements for stability, static stability and dynamic stability. For static stability, it just requires that the center of gravity $(\mathrm{CG})$ is within robot support area. But this case assumes robot moves very slowly so that the moments from reciprocating links can be neglected and the $\mathrm{CG}$ is consistent with center of pressure (CP). For dynamic stability, CP may be different from CG. Because it considers dynamic effects of the robot, the CG may lie outside of the support region. Most biped walking planning has been achieving dynamic stability. A popular method called Zero Moment Point (ZMP) [13, 14] has been used as a criterion of walking stability. The ZMP is defined as the point on the ground where the moment causes the ground reaction forces equal to zero. It can also been taken as the CG. Only if the ZMP resides in the support region, the biped robot can walk avoiding falling. Any biped walking planning has to abide this basic principle.

Another basic requirement for trajectory planning is to achieve smooth walking pattern for biped robots [3, 6, 9]. In this regard, the joint trajectories should be characterized as being continuous for both first and second order derivatives. The first-order derivative continuity guarantees the smoothness of joint angular velocity while the second-order derivative continuity guarantees the smoothness of acceleration or torque of the joint. Furthermore, it can be proved that derivative continuity in the Cartesian space can guarantee the first and second derivative continuity in the joint space, and vice versa.

In this paper, we present a third-order spline interpolation based trajectory planning method aimed to achieve smooth biped swing leg trajectory by reducing the instant velocity change which occurs near the time of collision of the biped swing leg with the ground. The impact effects of biped contact with the environment were firstly studied by Zheng and Memami [15]. They showed that the impact may cause large internal impulsive forces in the body, the biped can control its initial posture in order to reduce the impulsive component of the internal constraint forces. They also proposed a linear velocity and nonlinear position feedback control method to slow down the biped motion to finally reach a static posture after hitting the ground. In this paper, we study the impact effects in biped landing on heels and toes by using the concept of spline interpolation [1] and zero moment points (ZMP) [13, 14]. This proposed method can guarantee both first-order and second- order derivative continuities at both start and end points of spline.

This paper is organized as follows. In Section 2, based on the analysis of bipedal walking cycle, the impact effects which occur at the time of the swing foot's heel touching with the ground are identified. The proposed spline based swing leg trajectory planning method is described in Section 3. Simulation and experimental results are presented in Section 4. Finally, conclusions and future work are given in Section 5. 


\section{BIPEDAL WALKING CYCLE AND IMPACT EFFECTS}

A complete walking cycle is composed of two phases: a double support phase and a single support phase. During the double support phase, both feet are in contact with ground, supporting leg changes from one to another. During the single support phase, one foot is stationary on the ground, the other foot swings from rear to front. The walking cycle is shown in Fig. 1, where Tc is the time period for one walking cycle, Td is the interval of double support phase. The right leg is drawn by solid line and is assumed as swing leg. This walking cycle starts at time Ts when left leg touch the ground, from $t=T s$ to $t=T s+T d$ is double support phase. From $\mathrm{t}=\mathrm{Ts}+\mathrm{Td}$ to $\mathrm{Ts}+\mathrm{Tc}$ is single support phase, the right leg swings from rear to front. At $t=\mathrm{Ts}+\mathrm{Tm}$, the right leg reach the highest point. The height and other parameters are shown in Fig. 2. Qf and Qb are the foot angle when the leg touches and leaves the ground respectively. When the leg leaves the ground, we name it leaving-point (LP), when it touches the ground, we name it touching-point (TP), when its toe touches with the ground, it is named fix-point (FP). For the right leg shown in Fig. 1, it is at $\mathrm{LP}$, when $\mathrm{t}=\mathrm{Ts}+\mathrm{Td}$, it is at TP when $\mathrm{t}=\mathrm{Ts}+\mathrm{Tc}$, it is at $\mathrm{TF}$ when $\mathrm{t}=\mathrm{Ts}+\mathrm{Tc}+\mathrm{Td}$.

A consecutive walking simulation is shown in Fig. 3. Based on the density variance in the Fig. 3, it can be concluded that during the double support phase the biped walks much slower than its single support phase. So there must exist instant velocity changes at the transition points (i.e. LP, TP and FP) between the single support and the double-support phases. From Fig. 1, it may have high acceleration value at $\mathrm{t}=\mathrm{Ts}$, $\mathrm{Ts}+\mathrm{Td}$ and $\mathrm{Ts}+\mathrm{Tc}$. At these points, the robot may be unstable.

In Fig. 2, the swing leg (right leg) trajectory is composed of two parts. The first part is from $t=T d+T d$ to Ts + Tm, which can be generated through 3D spline interpolation, the interpolation points are positions of the right leg at $\mathrm{t}=\mathrm{Ts}+\mathrm{Td}, \mathrm{Ts}+\mathrm{Tm}$ and $\mathrm{Ts}+\mathrm{Tc}$. These points are predefined. The second part is the trajectory of right leg, from $\mathrm{t}=\mathrm{Ts}$ to $\mathrm{Ts}+\mathrm{Td}$, and from $\mathrm{Ts}+\mathrm{Tc}$ to $\mathrm{Ts}+\mathrm{Tc}+\mathrm{Td}$. Both of trajectories are arc which cannot be changed. Our focus of this work is to get a trajectory which can guarantee both the first order and second derivative continuities at the transiting point between the two trajectories. We also find that after FP, the right foot touches with the ground to wait for another cycle. Please note that at this time the CG has moved to the right foot and the heel of the right foot is an unpowered degree-of-freedom (DOF). Hence, the instant velocity change at this point is unavoidable.

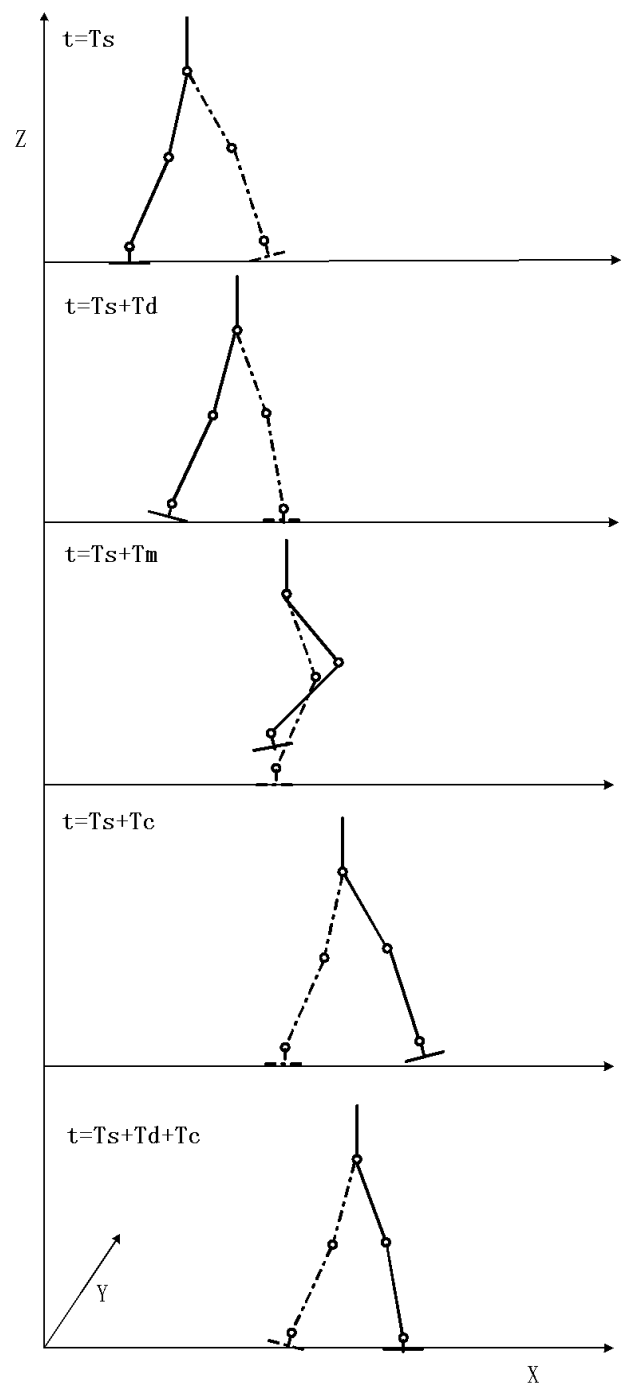

Fig.1. Walking cycles

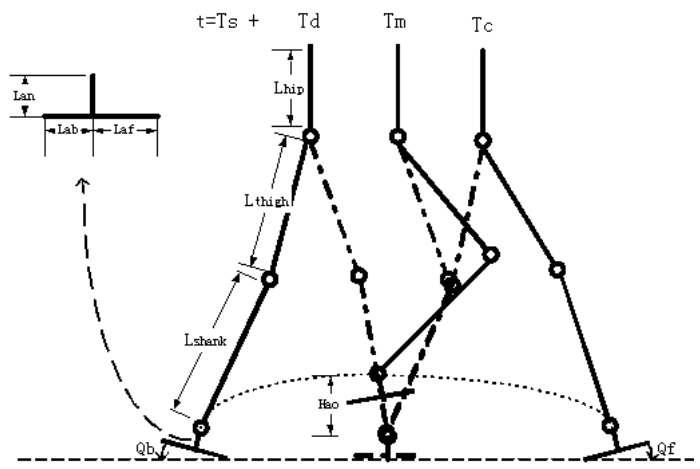

Fig.2. Trajectory parameters 


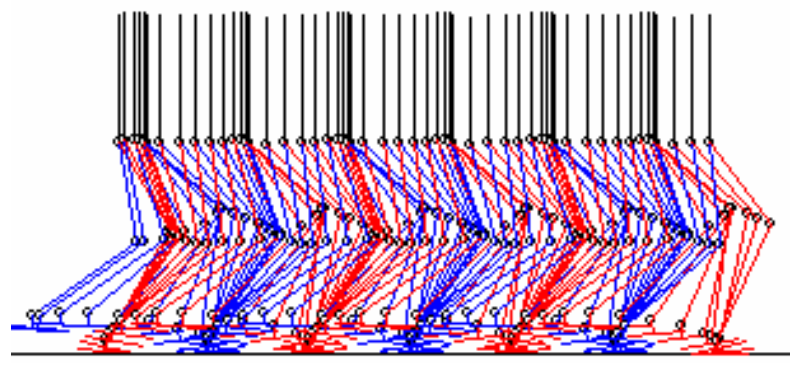

Fig.3. Consecutive walking simulation

\section{CONTINUOUS SWING LEG TRAJECTORY}

To achieve the continuity between the trajectories in single support and double support phases, we propose the following trajectory planning method based on the third-order spline [1].

$$
\begin{aligned}
& \text { Hao }=a_{0} T_{m}^{3}+b_{0} T_{m}^{2}+c_{0} T_{m}+d_{0} \\
& z_{1}\left(T_{d}\right)=a_{0} T_{d}^{3}+b_{0} T_{d}^{2}+c_{0} T_{d}+d_{0} \\
& \text { Hao }=a_{1} T_{m}^{3}+b_{1} T_{m}^{2}+c_{1} T_{m}+d_{1} \\
& z_{2}\left(T_{c}\right)=a_{1} T_{c}^{3}+b_{1} T_{c}^{2}+c_{1} T_{c}+d_{1} \\
& 3 * a_{0} * T_{m}^{2}+2 * b_{0} * T_{m}+c_{0}=3 * a_{1} * T_{m}^{2}+2 * b_{1} * T_{m}+c_{1}
\end{aligned}
$$

$$
\begin{aligned}
& 6 * T_{m}+2 * b_{0}=6 * T_{m}+2 * b_{1} \\
& 3 * a_{1} * T_{c}^{2}+2 * b_{1} * T_{c}+c_{1}=k_{2} * L a b * \cos (Q f) \\
& 6 * a_{1} * T_{c}+2 * b_{1}=-k_{2}^{2} * L a b * \sin (Q f) \\
& 3 * a_{0} * T_{0}^{2}+2 * b_{0} * T_{d}+c_{0}=k_{1} * L a f * \cos (Q b) \\
& 6 * a_{0} * T_{d}+2 * b_{0}=-k_{1}^{2} * L a f * \sin (Q b) \\
& z_{1}(t)=L a f * \sin \left(Q b+k_{1} *\left(t-T_{d}\right)\right) \\
& z_{2}(t)=L a b * \sin \left(Q f+k_{2} *\left(t-T_{c}\right)\right)
\end{aligned}
$$

The Equations (1) to (6) fulfill the spline conditions. $a_{i}, b_{i}, c_{i}$ and $d_{i}(i=0,1)$ are coefficients of the spline line. Equations (11) and (12) are trajectories in $\mathrm{z}$ axis from $\mathrm{t}=\mathrm{Ts}$ to $\mathrm{t}=\mathrm{Ts}+\mathrm{Td}$ and from $\mathrm{t}=\mathrm{Ts}+\mathrm{Tc}$ to $\mathrm{t}=\mathrm{Ts}+\mathrm{Tc}+\mathrm{Td}$, which are two arcs in XOZ plane, $\mathrm{k}_{1}$ and $\mathrm{k}_{2}$ are foot angular velocity near the LP and TP. Equations (7), (8), (9) and (10) guarantee the first and second order derivative continuities of the trajectories at TP and LP. From the Equations (1) to (12), we can derive the trajectory of swing leg in Z-axis. The trajectory in X-axis can be obtained in the same way.

From the Equations (1) to (12), once the three interpolation points are defined, the angular velocity of the ankle, near the collision, can be obtained. This velocity may be considered to perform a function as an elastic surface, which can absorb impact effects at the time of collision between the toe and the ground.

\section{EXPERIMENTAL RESULTS}

In this section, we apply the proposed trajectory planning method to a biped robot developed at Singapore Polytechnic. The simplified three-dimensional link structure of the biped robot is shown in Fig. 4. Its total mass is about $2.5 \mathrm{~kg}$. The height is $60 \mathrm{~cm}$. Each leg has five active DOF of which two DOF are available at the hip, one at the knee and two at the ankle joint. In order to show the effectiveness of the proposed swing leg trajectory, we compare two trajectories, one is gotten from the proposed trajectory planning method as described in Equations (1) to (12), the another one is gotten from Matlab spline function. The parameters of the walking cycle is given in Table I $(\mathrm{Hao}=5 \mathrm{~cm}, \mathrm{Qf}=\mathrm{Qb}=0.5 \mathrm{rad})$. The parameters of the biped robot used in the following simulation are shown in Table II.

Based on the parameters shown in Tables I and II, The swing leg trajectory in z-axis generated by the Matlab Spline function is shown in Fig. 5, its corresponding ZMP trajectory is illustrated in Fig. 6. From Fig. 6, it can be seen that there are some instant changes in the ZMP trajectory at those black points, which are resulted from the discontinuities of the trajectory at TP and FP (shown in Fig. $5)$.

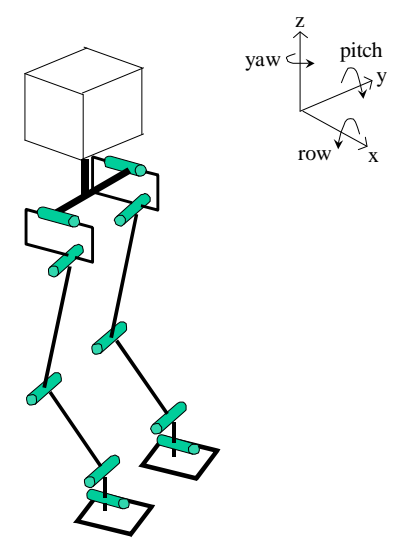

Fig.4.. The link structure of the biped robot.

TABLE I. PARAMETERS OF WALKING CYCLE

\begin{tabular}{|l|l|l|l|}
\hline Time & $\mathrm{Td}$ & $\mathrm{Tm}$ & $\mathrm{Tc}$ \\
\cline { 2 - 4 } Point ( s) & 0.15 & 0.5 & 0.9 \\
\hline
\end{tabular}




\begin{tabular}{|l|l|l|l|l|l|}
\hline $\mathrm{L}_{\text {hip }}$ & $\mathrm{L}_{\text {thigh }}$ & $\mathrm{L}_{\text {shank }}$ & $\mathrm{L}_{\mathrm{an}}$ & $\mathrm{L}_{\mathrm{ab}}$ & $\mathrm{L}_{\mathrm{af}}$ \\
\hline $20 \mathrm{~cm}$ & $12 \mathrm{~cm}$ & $12 \mathrm{~cm}$ & $7 \mathrm{~cm}$ & $8 \mathrm{~cm}$ & $8 \mathrm{~cm}$ \\
\hline
\end{tabular}

Using the same parameters as Tables I and II and solving the proposed Equations (1) to (12), we can derive another spline. Its coefficients and angular velocity are shown in Tables III and IV. Its swing leg trajectory is given in Fig. 7. Fig. 8 shows its ZMP trajectory.

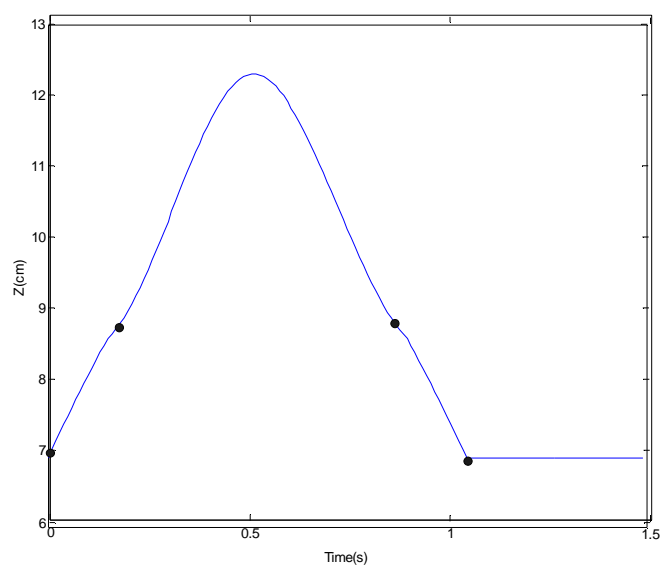

Fig.5. Swing leg trajectory

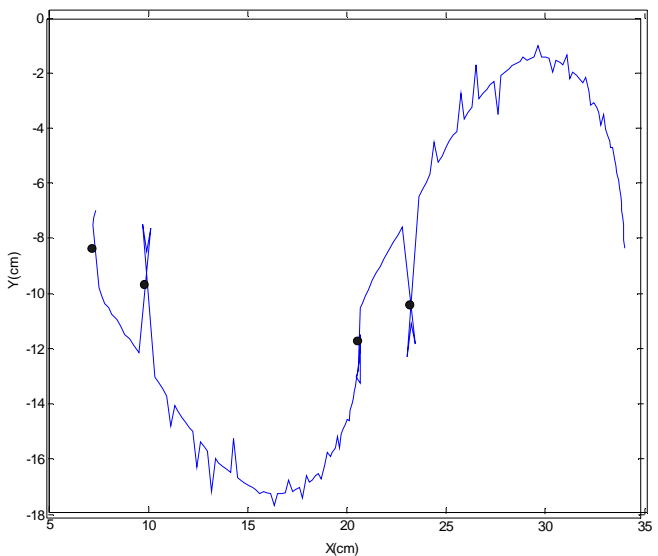

Fig.6. The ZMP trajectory (the black points represent fluctuation points.)

TABLE III. COEFFICIENTS OF THE PROPOSED SPLINE

\begin{tabular}{|l|l|l|l|}
\hline $\mathrm{a}_{0}$ & $\mathrm{~b}_{0}$ & $\mathrm{c}_{0}$ & $\mathrm{~d}_{0}$ \\
\hline-0.0002 & 0.0184 & -0.0986 & 3.09 \\
\hline $\mathrm{a}_{1}$ & $\mathrm{~b}_{1}$ & $\mathrm{c}_{1}$ & $\mathrm{~d}_{1}$ \\
\hline 0.0002 & -0.0433 & 2.9899 & -48.385 \\
\hline
\end{tabular}

\begin{tabular}{|l|l|l|}
\hline $\begin{array}{l}\text { Angular } \\
\text { Velocity }\end{array}$ & $\mathrm{k}_{1}$ & $\mathrm{k}_{2}$ \\
\cline { 2 - 3 }$(\mathrm{rad} / \mathrm{s})$ & 0.3 & -0.3 \\
\hline
\end{tabular}

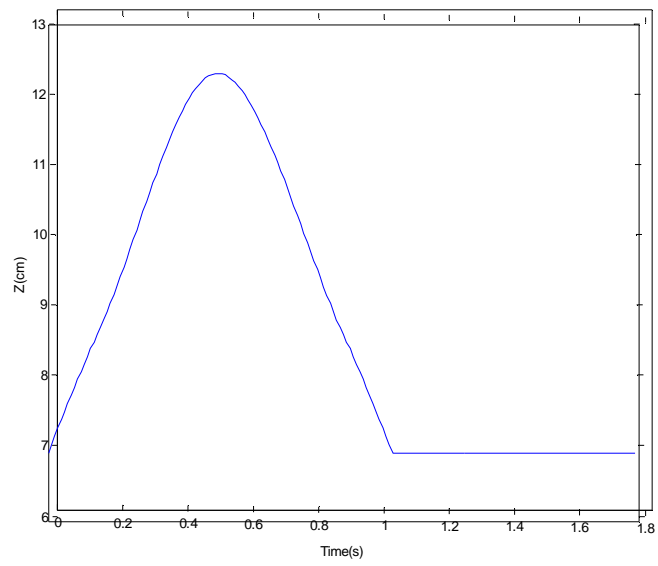

Fig.7. The proposed swing leg trajectory

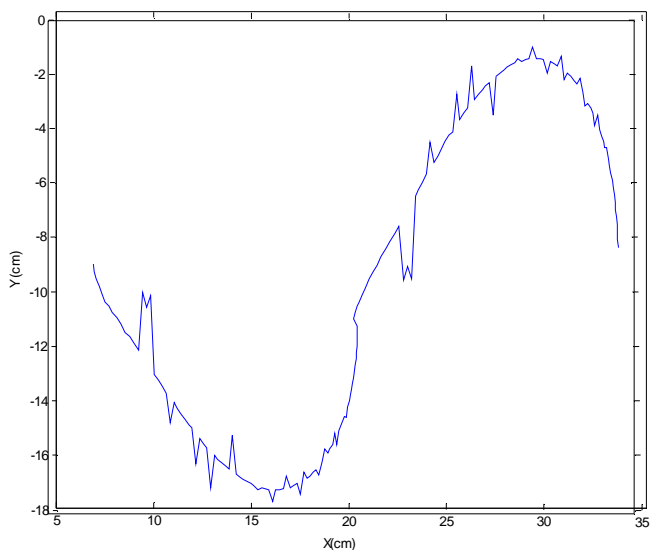

Fig.8. The ZMP trajectory generated by the proposed method

As shown in Fig 8, our proposed trajectory can eliminate the ZMP fluctuation at the TP. Furthermore, it can also relieve the fluctuation at the FP.

Fig. 9 shows the swing leg trajectory in both $\mathrm{z}$ axis and $\mathrm{x}$ axis. Based on the proposed swing leg trajectory planning approach, the joint angle trajectories (hip, knee and ankle) are derived from the position of the joints in the Cartesian space by inverse kinematics equation. These angle trajectories are shown in Fig. 10. 


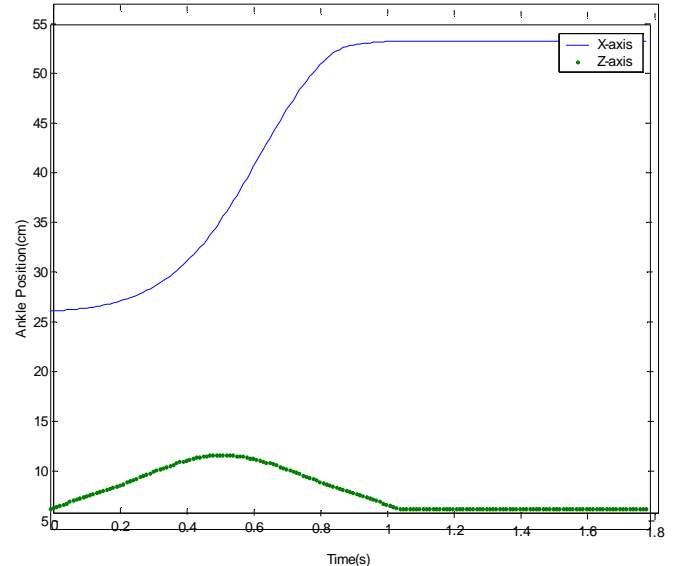

Fig.9. Swing leg trajectories in Z-axis and $\mathrm{X}$-axis

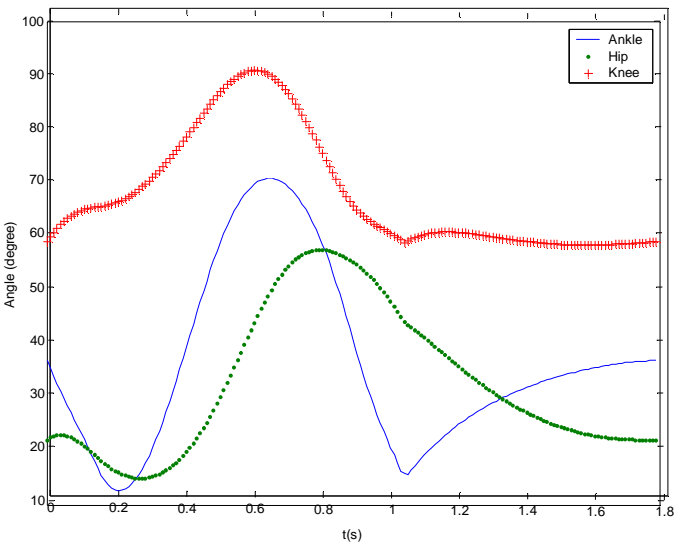

Fig.10. Joint angle trajectories

To prove the effectiveness of the proposed swing leg trajectory planning method, we have implemented the hip, knee and ankle joint angle trajectories (Fig. 10) in our newly developed soccer-playing humanoid robot, Robo-Erectus (Fig. 11).

The Robo-Erectus is a low-cost autonomous humanoid robot being developed at the ARICC (Advanced Robotics and Intelligent Control Centre) in the School of Electrical and Electronic Engineering, Singapore Polytechnic. The current challenge for the Robo-Erectus project is to develop a team of low-cost soccer-playing humanoid robots that are capable of walking, turning, crouching, and kicking a football past a goalkeeper into the goal $[8,12]$. The ultimate aim of this project is to develop and productize a low-cost humanoid robot so that people are able to build humanoids easily and cheaply and the humanoids are accessible to many people and can better fit in with human society.

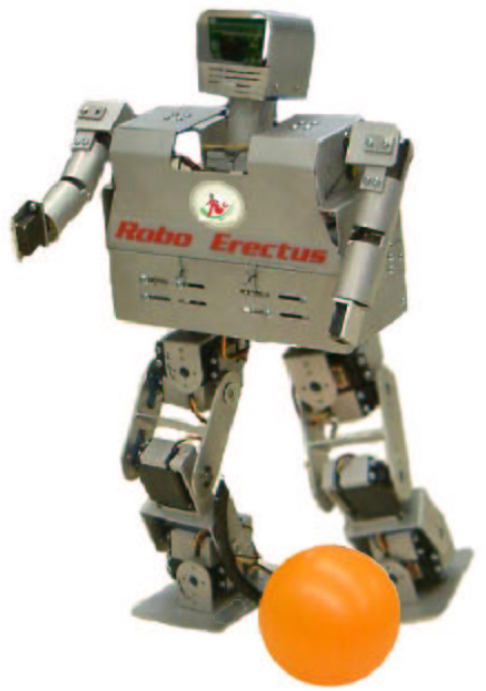

Fig. 11 The soccer-playing humanoid robot, Robo-Erectus, developed at Singapore Polytechnic (www.robo-erectus.org)

\section{CONCLUSIONS AND FUTURE WORK}

In this paper, we propose a third-order spline interpolation based trajectory planning method to plan a smooth biped swing leg trajectory by reducing the instant velocity change which occurs at the time of collision of the biped swing leg with the ground. We demonstrate that the impact effects can be avoided at the time of the swing foot's heel touching with the ground. The proposed biped trajectory planning method has been successfully tested on our newly developed soccer-playing humanoid robot, Robo-Erectus.

It can be seen that the proposed trajectory for swing leg can eliminate fluctuation at TP (touching-point), however the fluctuation at FP (fix-point) is unavoidable due to the discontinuities at the moment of the biped transition between the single support and double support phases. How to reduce the fluctuation at FP is another challenging issue for this research. To reduce the instant velocity change at FP, we have to let the foot angular velocity $\left(\mathrm{k}_{1}\right.$ and $\left.\mathrm{k}_{2}\right)$ as slow as possible. However, this will slow down the biped walking in the double support phase. We will have to compromise these two factors based on the requirements in humanoid planning and control.

Humans have remarkable learning capability in the uncertain and unpredictable environments. How to make use of learning $[16,17,18]$ and soft computing techniques [7] to further improve biped trajectories will be our future research. 


\section{ACKNOWLEDGEMENTS}

This project is supported by the Singapore Polytechnic R\&D fund (11-27801-36-R035) and the Singapore Tote Board fund. The authors would like to thank researchers and students at the ARICC (Advanced Robotics \& Intelligent Control Centre) in the School of Electrical and Electronic Engineering, Singapore Polytechnic, for their kind support in the humanoid hardware and software development.

\section{REFERENCES}

[1] C. de Boor, A Practical Guide to Splines, Springer-Verlag, 1978.

[2] J.J. Craig, Introduction to Robotics: Mechanics and Control, $\quad 2^{\text {nd }}$ Edition, Addison-Wesley, 1989.

[3] R.D. Eric and D.H. Robert, "Towards Smooth Bipedal Walking," in Proc. IEEE Int. Conf. Robotics and Automation, San Diego, CA, May 1994, pp. 2489-2494.

[4] T. Furuta, T. Tawara, Y. Okumura, M. Shimizu, K.Tomiyama, "Design and construction of a series of compact humnoid robots and development of biped walk control strategies," Robotics and Autonomous Systems, Vol. 37, 2001, pp.81-100.

[5] Q. Huang, K. Yokoi, S. Kajita, K. Kaneko, H. Arai, N. Koyachi, and K. Tanie, "Planning Walking Patterns for a Biped Robot," IEEE Trans. Robot. Automat, Vol. 17, June 2001, pp.280-289.

[6] D. Ito, T. Murakami and K. Ohnishi, "An approach to generation of smooth walking pattern for biped robot," in Proc. of the $7^{\text {th }}$ Workshop on Advanced Motion Control, Maribor, Slovenia, 2002, pp.98-103.

[7] D. Katic and M. Vukobratovic, "Intelligent soft-computing paradigms for humanoid robots," in Proc. IEEE/RSJ Int. Conf. on Intelligent Robots and Systems, EPFL, Lausanne Switzerland, Oct. 2002, pp. 2533-2538.

[8] H. Kitano and M. Asada, "The RoboCup humanoid challenge as the millennium challenge for advanced robotics," Advanced Robotics, Vol. 13, No. 8, 2000, pp.723-736.
[9] J.H. Park, "Fuzzy-logic zero-moment-point trajectory generation for reduced trunk motions of biped robots," Fuzzy Sets and Systems, Vol. 134, 2003, pp.189-203.

[10] C.-L. Shih, "Gait Synthesis for a biped robot," Robotica, Vol. 15, 1997, pp.599-607.

[11] C.-L. Shih, "Ascending and Descending Stairs for a Biped Robot," IEEE Trans. System, Man, and Cybernetics, Vol. 29, May 1999, pp.255-268.

[12] Z. Tang, C. Zhou and Z. Sun, "Gait synthesizing for humanoid penalty kicking," the 3rd International DCDIS Conference on Engineering Applications and Computational Algorithms, Guelph, Ontario, Canada, May 15-18, 2003 (accepted).

[13] M. Vukobratovic, D. Juricic, "Contributions to the synthesis of biped locomotion," IEEE Trans. On Bio\&Eng, Vol. BME-16, No. 1, Jan 1969, pp.1-6.

[14] M. Vukobratovic and B. Borovac, D. Surla, D. Stokic, Biped Locomotion: Dynamics, Stability, Control and Application, Springer-Verlag, Berlin- Heidelberg, 1990.

[15] Y. Zheng, and H. Hemani, "Impact Effects of Biped Contact with the Environment," IEEE, Trans. Systems, Man and Cybernetics, Vol. SMC-14, No. 3, May 1984, pp.437-443.

[16] C. Zhou, "Neuro-fuzzy gait synthesis with reinforcement learning for a biped walking robot," Soft Computing, Vol.4, No.4, 2000, pp.238-250.

[17] C. Zhou, "Robot learning with GA-based fuzzy reinforcement learning agents," Information Sciences, vol.145, 2002, pp.45-68.

[18] C. Zhou and Q. Meng, "Dynamic balance of a biped robot using fuzzy reinforcement learning agents," Fuzzy Sets and Systems, Vol.134, No.1, 2003, pp.169-187. 